\title{
PROCESSO DE DESENVOLVIMENTO DE PRODUTOS INTEGRADO COM A METODOLOGIA DE AVALIAÇÃO DE PRONTIDÃO TECNOLÓGICA: PROPOSTA PARA UM CENTRO DE TECNOLOGIA EM NANOMATERIAIS
}

Raissa Guerra Resende (raissaguerraresende@gmail.com.br) - Universidade Federal de Minas Gerais

Raoni Barros Bagno (rbagno@dep.ufmg.br) - Universidade Federal de Minas Gerais

Glaura Goulart Silva (glaurasilva@ctnano.com.br) - Centro de Tecnologia em Nanomateriais

Lucas Campos Moreira (lucas.campos@iebt.com.br) - IEBT

Ana Maria Azevedo Amaral (anamaria.amaral@iebt.com.br) - IEBT

Paulo Vítor Guerra (paulo.guerra@iebt.com.br) - IEBT

\begin{abstract}
RESUMO
O uso de um Processo de Desenvolvimento de Produtos (PDP) tem sido considerado na literatura como fator de sucesso para o desenvolvimento de inovações nas organizações. Não obstante, estudos recentes argumentam que modelos consolidados tomados em suas formas tradicionais são pouco apropriados para projetos com grandes incertezas e riscos. Diante disso, este estudo faz uma proposta de PDP para um Centro de Tecnologia em Nanomateriais, conduzido sob o arcabouço da literatura de desenvolvimento de novos produtos e tecnologias e das possibilidades de combinação com a abordagem de Technology Readiness Levels. À luz das recomendações da pesquisa-ação, foi construido um modelo de processo para a organização, que contribuiu para: (i) acompanhamento e proposições de ações específicas para projetos com diferentes niveis de maturidade; (ii) melhor identificação e direcionamento da atuação para novas oportunidades de mercado e interfaces entre frentes de pesquisas. A proposta contribui com o debate de processos de inovação em contextos de alta complexidade tecnológica ao integrar aspectos típicos de P\&D com questões tradicionais de PDP. O estudo também ilustra possibilidades de diálogo entre os aspectos de flexibilidade e padronização de processos em ambientes de alta incerteza, opções comumente percebidas como dicotômicas.
\end{abstract}

Esta publicação tem apoio financeiro da FAPEMIG.

Palavras chave: Inovação Tecnológica; Gestão do Desenvolvimento de Produto; Avaliação de Prontidão Tecnológica; Nanotecnologia.

Área: Modelos de processo para o desenvolvimento de produtos e serviços

\section{INTRODUÇÃO}

A nanotecnologia é uma área emergente da ciência, na qual segundo a US National Nanotechnology Initiative (2007, p. 5), fenômenos únicos permitem novas aplicações. Esta possui caráter transdisciplinar sendo reconhecida por seu grande potencial socioeconômico a ser explorado em várias cadeias produtivas. Contudo, segundo Piscopo et al. (2013), o setor de nanotecnologia no Brasil ainda se encontra bastante incipiente, necessitando de suporte nas esferas pública e privada não somente para o Desenvolvimento Tecnológico (DT), mas também para a comercialização dos produtos e serviços resultantes de sua aplicação. 
Dentre as diversas formas de contribuição para o desenvolvimento de nanotecnologias, está o suporte às questões internas vinculadas à Gestão do Desenvolvimento de Produtos (GDP) das organizações, o que pode ser determinante para o sucesso (ou fracasso) dos negócios. Tal suporte justifica-se dado que as organizações se deparam com desafios e particularidades decorrentes da nova base tecnológica sobre a qual se apoiam. O que implica em altos níveis de incerteza - tecnológica e mercadológica - permeando os negócios de empresas desse setor. Ademais, percebe-se a concomitância entre os desafios de se desenvolver novas tecnologias juntamente com suas respectivas aplicações. Tal contexto é um terreno fértil para o desenvolvimento teórico e aplicado de GDP.

Diante disso, este estudo se debruça sobre um esforço realizado ao longo de nove meses para construção de uma proposta de PDP para um Centro de Tecnologia (CT) em Nanomateriais. Tal esforço foi conduzido sob o arcabouço da literatura de gestão de desenvolvimento de novos produtos e de tecnologias e também das possibilidades de combinação destes com a abordagem de avaliação dos Technology Readiness Levels (TRLs). A estratégia metodológica de campo, feita à luz das recomendações da pesquisa-ação, permitiu a construção coletiva das respostas e soluções para os desafios identificados junto à equipe do CT, resultando em um processo adaptado às particularidades dos projetos da organização e voltado à gestão da inovação de forma estruturada do ponto de vista dos processos, decisões e atribuições nela envolvidos.

O presente texto encontra-se estruturado da seguinte maneira: inicialmente são apresentados os referenciais teóricos que deram base para a confecção do trabalho. Em seguida é retratada a metodologia para o passo-a-passo de desenvolvimento do projeto. $\mathrm{Na}$ terceira parte, além de contextualizar o CT, são apresentados, analisados e discutidos os resultados do trabalho. Por fim, se tem uma breve conclusão abordando os próximos passos e as contribuições esperadas.

\section{REFERENCIAL TEÓRICO}

\subsection{Os papeis e desafios da gestão tecnológica}

O termo "tecnologia" é definido pelo Webster's Seventh Collegiate Dictionary como a totalidade dos meios utilizados para prover os objetos necessários para o sustento e conforto humano. Sob esse aspecto, pode-se afirmar que a tecnologia não consiste no produto final em si, mas sim, ela contribui ou é inserida durante o processo de formação do produto final. É quando o último chega as mãos do consumidor que se constata o valor da(s) tecnologia(s) incorporadas, a partir de seu potencial de utilização e aplicação vistos em sua exploração comercial. Essa definição contribui para conceituar o "(...) desenvolvimento de um produto como um processo de transformação de ideias, dados de mercado e tecnologias (já existentes ou novas), em novos conhecimentos para produção comercial" (SILVA, 2002, p. 2). Portanto, é possível concluir que tal processo pode envolver também etapas referentes ao desenvolvimento de novas tecnologias.

O DT é, por natureza, formado por projetos de alto risco com muitos elementos desconhecidos e incertezas técnicas (COOPER, 2007). O que influencia para que, no contexto típico de empresas industriais, os projetos majoritariamente advenham de atualizações, modificações e melhorias de produtos existentes (COOPER, 2005). Dessa forma, tal contexto fundador e central nas discussões predominantes da GDP, se distancia dos desafios típicos de desenvolvimento de novas tecnologias, que frequentemente resultam na fundação de uma plataforma para uma nova linha de produtos ou na base de um novo negócio. Portanto, tais elementos contribuem para que, segundo COOPER (2007), os projetos de desenvolvimento de novas tecnologias sejam cada vez mais raros no portfólio de projetos das organizações. 


\subsection{Processos de desenvolvimento de produtos e tecnologias}

A necessidade de um Processo gerencial de desenvolvimento de produtos tem sido largamente considerada na literatura como fator de sucesso para o desenvolvimento de inovações nas organizações. Sua criação justifica-se quando se tem a expectativa de recorrência regular do processo. Dentre vários estudos e modelos referência na área de Desenvolvimento de Produtos (DP) que se relacionam a tal abordagem, ressalta-se: Pugh (1991) que apresenta o modelo total design que tem como foco as incertezas técnicas no Processo de Desenvolvimento de Novos Produtos; Cooper (1990) que confeccionou um modelo marcado por uma série de fases que englobam da ideia até o lançamento do produto, sendo que, o avanço do projeto entre as fases é marcado pela análise de pontos de decisão, denominados stage gates; Thomas (1993) faz sua contribuição a partir um modelo que traz o conceito de que cada estágio do PDP possui diferentes dimensões - ideias, conceitos, protótipos, produtos e programas de marketing - no qual cada produto específico demanda uma dinâmica de maturidade distinta para cada dimensão; o modelo de Rozenfeld et al. (2006), no qual os estágios do desenvolvimento são agrupados em 3 macroetapas distintas, que englobam desde o planejamento estratégico até a descontinuação do produto.

Salerno et al. (2015) indicam que os PDPs tradicionais são focados em grandes empresas, com departamentos de Pesquisa e Desenvolvimento (P\&D) estabelecidos e possuem projetos que alocam uma quantidade significativa de tempo e recursos financeiros. Via de regra, segundo eles, modelos de PDPs comumente aceitos baseiam-se em produtos tangíveis, montados e de estrutura complexa, de média ou alta tecnologia, produzidos em altos volumes para serem estocados e possuem estratégia de negócio B2C (Business to Consumer). Ademais, esses modelos geralmente consideram o processo de inovação como uma sequência linear composta por fases pré-definidas: geração e seleção de ideias, desenvolvimento e lançamento para o mercado (SALERNO et al., 2015).

Não obstante, autores como Salerno et al. (2015) atentam-se para o fato de que modelos consolidados de PDP, tomados em suas formas tradicionais, não são diretamente aplicáveis a qualquer realidade. Por exemplo, esses não abrangem projetos envolvendo inovações radicais que possuem características distintas, como altos níveis de incertezas e complexidades (SALERNO et al., 2015). O debate tem fomentado o desenvolvimento de abordagens complementares ou mesmo alternativas, como a de flexibilização e abertura dos processos de inovação, modelos de Fuzzy Front End (FFE) ou avaliação dos níveis de maturidade tecnológica.

A rigor, há autores que tratam o DT como processo preliminar ou mesmo etapas preliminares ao DP tradicional, trazendo peculiaridades que devem ser consideradas, mas não são suficientes para desvincular ambos os processos. Como exemplos, Roberts (1988) apresenta um modelo focado no DT com etapas distintas e diferentes tipos de atividades e decisões; Cooper (2007) traz uma abordagem direcionada para o conteúdo das etapas e questões à serem respondidas no processo de DT; e Cheng et al. (2007), com foco nas empresas de base tecnológica de origem acadêmica, retratam o Processo de Planejamento Tecnológico para orientar a incorporação da tecnologia em produtos e serviços para o mercado.

Contudo, alguns estudos, principalmente na linha do FFE, consideram a dinâmica do DT como distinta o suficiente para se justificar como um processo à parte do DP. O conceito de Front End emerge no contexto em que segundo Khurana e Rosenthal (1998), ideias de novos produtos necessitam de ter um suporte adequado antes de serem formalmente desenvolvidas, sendo o Front End o momento que compreenderá as atividades para este fim. O Front End é também chamado de FFE por diversos autores, como Belliveau, Griffin, Somermeyer (2004), dada à natureza experimental do trabalho e incerta em relação aos resultados. Entretanto, tais 
características podem estar presentes também no DT, justificando sob um certo ponto de análise, abordagens que retratam o DT dentro dessa perspectiva, como aquelas apresentadas por Khurana e Rosenthal (1998) e Koen et al. (2002).

\subsection{Technology Readiness Assessment}

A utilização de uma metodologia para a avaliação da maturidade de uma tecnologia, auxiliando na identificação dos elementos e processos necessários ao seu desenvolvimento para o alcance de um determinado nível de maturidade, pode contribuir para a redução das incertezas técnicas e riscos relativos ao DT. Com essa finalidade, foi escolhida a abordagem de avaliação da prontidão tecnológica - Technology Readiness Assessment (TRA) - a partir de diferentes níveis de maturidade tecnológica - Technology Readiness Levels (TRLs). DoE (2011) relata que a metodologia TRA pode colaborar para: (i) a identificação de gaps em testes; (ii) demonstração e conhecimento sobre o atual nível de risco da tecnologia; (iii) realização do direcionamento dos passos necessários para o projeto alcançar um determinado nível de maturidade; (iv) identificação de riscos tecnológicos necessários de serem geridos com atenção ou com recursos adicionais; e (v) aumento da transparência na tomada de decisões a partir da identificação das tecnologias mais imaturas e que possuem maiores riscos.

O TRA é utilizado em diversos setores industriais (como aeroespacial, energia, defesa, petróleo e gás), sendo que sua aplicação deve estar alinhada à realidade do setor em questão, necessitando de adaptações e personalização para cada contexto. Como consequência, aplicações distintas usualmente utilizam diferentes definições para os TRLs. Entretanto, mesmo levando em consideração suas peculiaridades, percebe-se que essas definições se mostram conceitualmente similares. Tendo como referência a aplicação da metodologia originalmente na National Aeronautics and Space Administration (NASA), a escala de maturidade tecnológica é composta por nove TRLs, sendo TRL 1 o mais baixo e imaturo e TRL 9 o mais alto e maduro. Cada nível possui uma descrição característica que retrata os elementos que devem ser desenvolvidos pelo projeto com o objetivo de alcançar esse nível de maturidade. Além dessa descrição, cada nível conta com um critério de saída, utilizado para determinar se a nova tecnologia satisfaz os vários aspectos de maturação que definem tal nível. Desse modo, segundo a EESA (2008) um determinado TRL somente é alcançado quando o critério de saída relativo a ele é satisfeito.

\section{METODOLOGIA}

A estratégia metodológica de campo para a confecção do PDP customizado às necessidades do Centro de Tecnologia em Nanomateriais (CTNano) da Universidade Federal de Minas Gerais (UFMG), foi feita à luz das recomendações da pesquisa-ação. O que proporcionou a construção coletiva das respostas e soluções para os desafios identificados junto à equipe do $\mathrm{CT}$, resultando em um processo adaptado às características dos projetos da organização e voltado à gestão da inovação de forma estruturada. A duração deste trabalho foi cerca de 9 meses, demandando da equipe executora cerca de 300 horas dedicadas ao estudo, confecção e implantação inicial do PDP.

A equipe executora foi composta pelo coordenador de operações, uma gestora de projetos e uma estagiária, que foram acompanhados e auxiliados por um consultor mais experiente no tema GDP. Além desses, o trabalho contou também com a orientação e auxílio de um professor universitário, referência em GDP e gestão de inovação tecnológica.

A equipe do CTNano envolvida no processo de confecção do PDP continha majoritariamente supervisores das frentes de pesquisa, seis ao todo, e a coordenadora delas, que também atua 
como vice coordenadora do Centro - possuindo então, uma visão sistêmica e transversal de todos os projetos. Cada supervisor envolvido era responsável por uma frente de pesquisa, com exceção de dois deles que eram responsáveis pela mesma frente. Os supervisores possuem background técnico na área de ciência dos materiais, sendo responsáveis por supervisionar e garantir a execução das atividades do projeto pelo corpo técnico. Já os coordenadores das frentes de pesquisa são pesquisadores com background também na área de ciência dos materiais, e com experiência comprovada na área da frente pela qual é responsável, sendo eles que definem as estratégias e metodologias de P\&D a serem executadas no DT.

A presença da equipe executora do projeto dentro da infraestrutura do CTNano facilitou a realização de reuniões de coleta de dados, validação do que estava sendo confeccionado e esclarecimento de dúvidas. As informações fornecidas eram analisadas para direcionar o planejamento de ações e a consequente tomada de decisões. O passo-a-passo simplificado de como se desenvolveram os estudos, pesquisas e intervenções realizadas consta na Figura 1.

Figura 1. Fluxograma do passo-a-passo do trabalho realizado. Fonte: Os autores (2017)

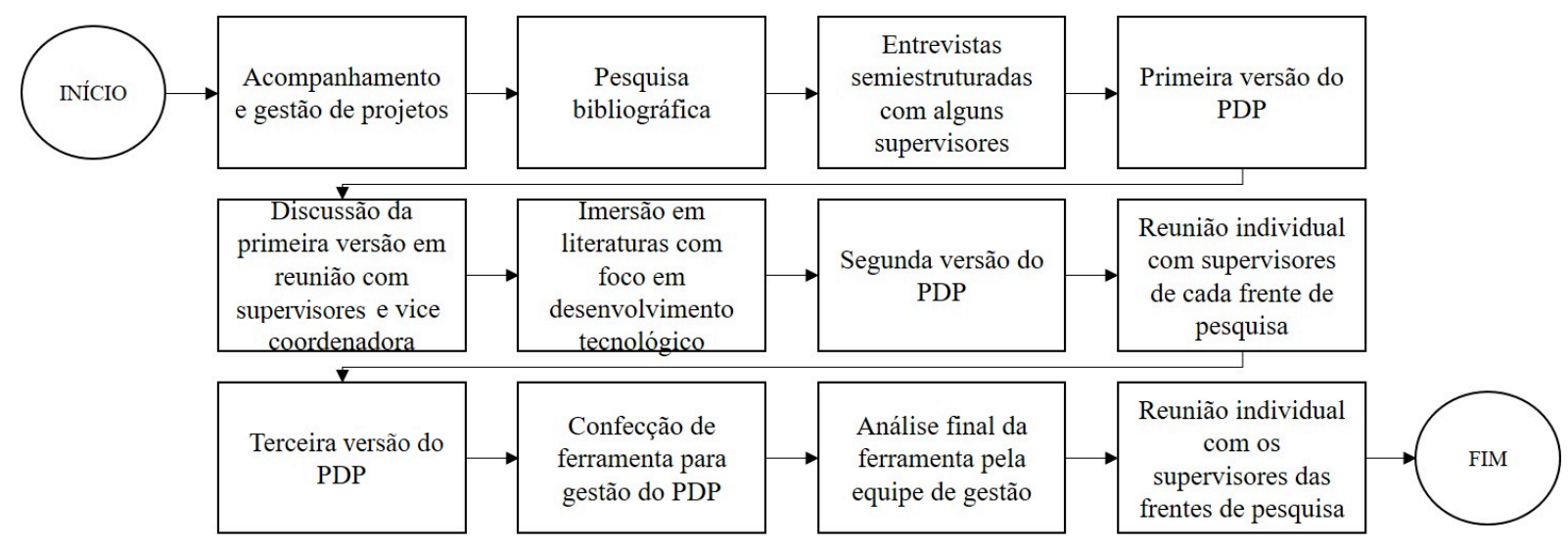

\section{RESULTADOS}

\subsection{Contextualizando o Centro de Tecnologia em Nanomateriais}

O CTNano está instalado no Parque Tecnológico de Belo Horizonte, tendo sido criado por meio da parceria entre agentes dos setores público e privado: Governo do Estado de Minas Gerais (SECTES e FAPEMIG), Fundo Tecnológico do Banco Nacional de Desenvolvimento Econômico e Social (FUNTEC-BNDES), e as Empresas Petrobras e InterCement. O objetivo do CTNano é o DT - de produtos, processos e serviços - a partir de nanomateriais, servindo de apoio à inovação em setores estabelecidos da indústria nacional e à geração de empresas de base tecnológica.

Para tal, são desenvolvidos, paralelamente, projetos em diferentes frentes de pesquisa, sendo a maioria desses vinculados a empresas/instituições parceiras técnicas e/ou financiadoras. Durante a realização desse trabalho, o CT contava com seis frentes de pesquisa e executava doze projetos, passíveis de ser localizados dentro da cadeia de valor da nanotecnologia, Figura 2.

Figura 2. Cadeia de valor da nanotecnologia. Fonte: Adaptado pelos autores a partir de Lux Research (2004)

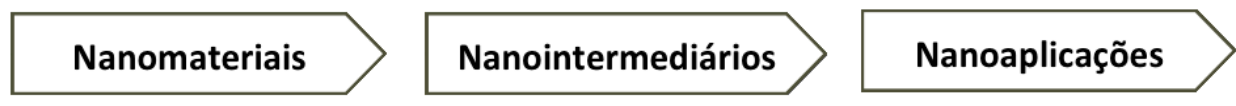

Segundo a Lux Research (2004) não há um mercado de nanotecnologia, mas sim uma cadeia de valor que vai desde os nanomateriais, nanointermediários até as nanoaplicações. Os 
projetos desenvolvidos no CTNano estão focados nos dois primeiros elos da cadeia. Sendo que sua estratégia visa atuação junto a empresas parceiras e/ou a geração de spinoffs no terceiro elo, para proporcionar a chegada do produto final, nanoaplicação, no mercado.

Por fim, vale enfatizar que o PDP confeccionado, considera o foco de atuação do CTNano dentro da cadeia de valor da nanotecnologia e, portanto, tem como objetivo central viabilizar a entrega de nanointermediários. Estes seriam os produtos finais do esforço de desenvolvimento da organização estudada, o que incorpora aspectos do próprio DT, não discerníveis, portanto, do produto final neste contexto. O intuito é que tal entrega se alinhe com as expectativas das empresas parceiras e/ou spinoffs consideradas.

\subsection{O Processo de Desenvolvimento de Produtos proposto}

A principal entrega do trabalho realizado no CTNano foi a confecção do PDP, Figura 3, vinculado a uma ferramenta para a gestão do desenvolvimento dos projetos. Tal ferramenta visava permitir um acompanhamento da evolução de cada projeto a partir das "respostas" aos gates e critérios de avanço definidos para cada etapa de desenvolvimento.

Figura 3. Visão geral da estrutura do Processo de Desenvolvimento de Produtos. Fonte: Os autores (2017)

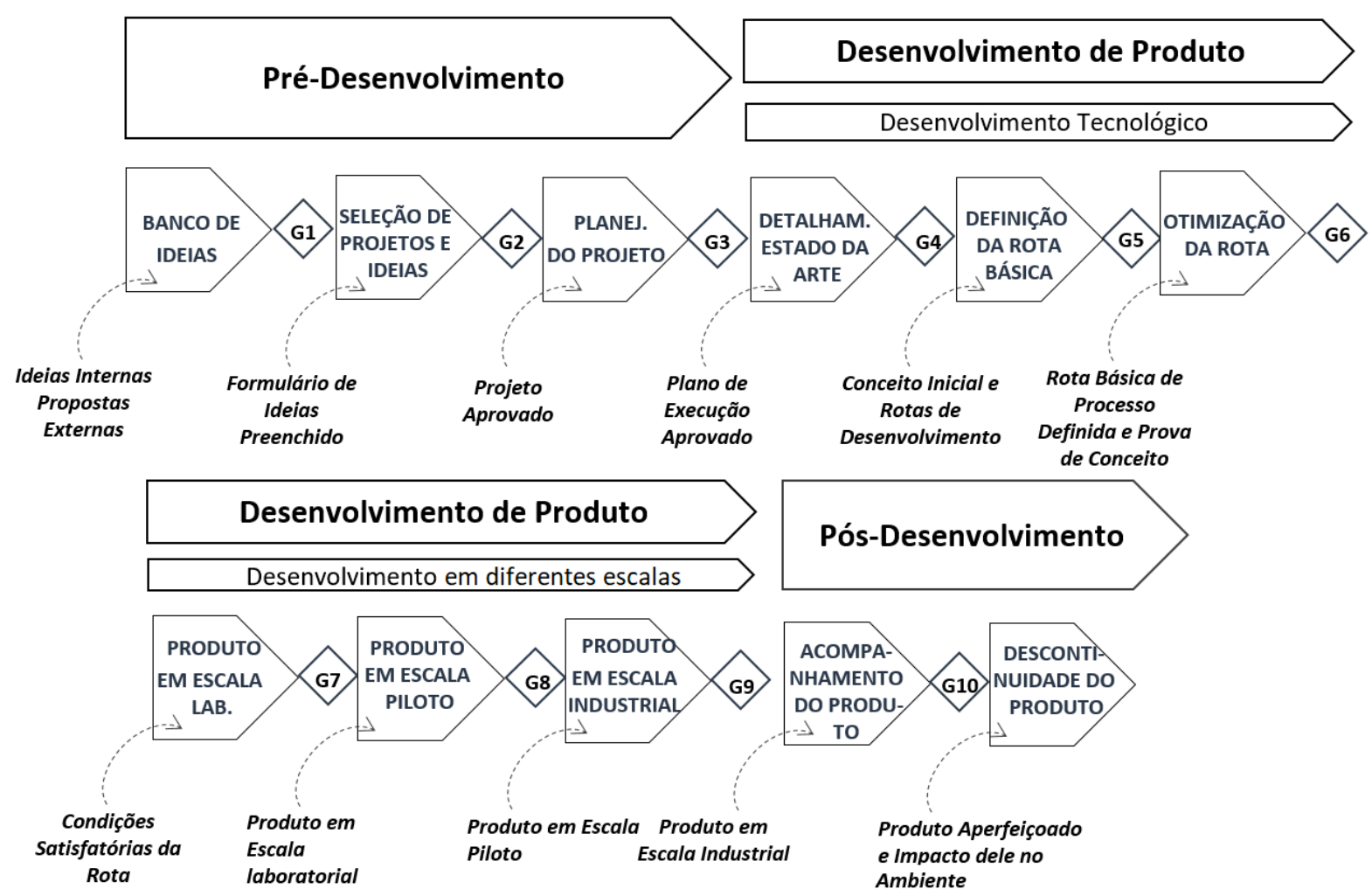

O PDP é formado por três macroetapas e onze etapas de desenvolvimento. A macroetapa de Pré-desenvolvimento de Produtos possui lógica de funil convergente, tendo como "input" as ideias internas e propostas externas de potenciais projetos, que serão avaliadas na etapa de Seleção de Projetos e Ideias. Tal etapa visa estabelecer um mix de projetos que maximizem o valor do portfólio do CTNano e que estejam alinhados com sua estratégia. Para isso, são utilizados critérios relativos a mercado, técnicos, financeiros, dentre outros. As ideias de projetos selecionadas passam para a etapa de Planejamento, na qual é confeccionado um plano de execução, que contará com as macro diretrizes do projeto. O plano aprovado consiste na saída dessa macroetapa, e também norteará a de Desenvolvimento de Produtos. 
Essa macroetapa inicia o DT, a partir da Revisão do Estado da Arte da Tecnologia, definição de possíveis rotas de desenvolvimento, seleção da rota básica e posteriormente a otimização da mesma. Em seguida, é iniciado o desenvolvimento do nanointermediário em diferentes escalas, alinhado aos interesses do parceiro ou spinoff responsável pela nanoaplicação. Isso irá ocorrer inicialmente a partir da validação dos parâmetros do processo de produção (como tempo de processamento, temperatura), por meio da integração da tecnologia ao produto em diferentes escalas: laboratorial, piloto e industrial. Vale ressaltar que a atuação do CTNano a partir da etapa de Produto em Escala Industrial irá ocorrer conforme alinhamento posterior com o parceiro. O que também se aplica à macroetapa de Pós-desenvolvimento.

Por fim, é válido ressaltar que a metodologia TRA se mostrou muito relevante na macroetapa de Desenvolvimento de Produtos em diversos momentos, como para a construção dos gates referentes aos aspectos técnicos do desenvolvimento. Isso pôde ser visto ao se correlacionar as etapas do PDP em construção, com os níveis de maturidade tecnológica presentes nas diferentes aplicações do TRA. Essa comparação permitiu a identificação de elementos das tecnologias, vinculados a determinado grau de maturidade que deveriam estar bem definidos e desenvolvidos, antes de se passar para outra etapa de desenvolvimento. Além disso, optou-se por ter maior flexibilidade em relação às respostas fornecidas nos gates (anteriormente tidas como "sim ou não"), com base na proposta do TRL Calculator de Nolte, Kennedy e Dziegiel (2003). Essa permite a definição de uma porcentagem de resolução às questões técnicas vinculadas a certos níveis de maturidade tecnológica. Tal abordagem convergiu com demandas apontadas pelos pesquisadores do CTNano, o que implicou em sua adoção.

A Figura 3 mostra a visão geral da estrutura do PDP, indicando também quais são os "inputs" de cada etapa de desenvolvimento. Já a Figura 4 descreve os gates estabelecidos para a avaliação do projeto na etapa de Detalhamento do Estado da Arte da Tecnologia, sem mostrar seu acompanhamento - o que também está presente na ferramenta construída. Conforme indicado na Figura 4, os gates foram classificados de duas formas distintas: quanto a sua natureza - técnico, mercado, Saúde, Meio Ambiente e Segurança (SMS), gestão - e quanto a tomada de decisão possível diante da resposta fornecida - GO/KILL/HOLD/RECYCLE. A primeira classificação, inspirada no TRL Calculator, objetiva assegurar a avaliação de todas as dimensões ao longo das etapas processo, enquanto a segunda indica a criticidade da tomada de decisão e direciona o que deve ser feito com o projeto depois de respondido o gate.

Figura 4. Gates da etapa do detalhamento do estado da arte da tecnologia. Fonte: Os autores (2016)

\begin{tabular}{|c|c|c|c|c|}
\hline \multicolumn{2}{|c|}{ Macroetapa } & \multirow{3}{*}{ 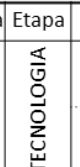 } & Classificação do Gate & GATE \\
\hline \multirow{10}{*}{ 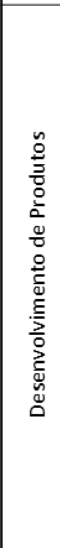 } & \multirow{10}{*}{ 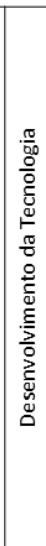 } & & Técnico Go/Recycle & $\begin{array}{l}\text { Foram realizados estudos exaustivos sobre o estado da arte considerando as potenciais áreas de aplicação (ex: } \\
\text { publicações, teses, dissertações, monitoramento tecnológico)? }\end{array}$ \\
\hline & & & Técnico Go/Kill/Hold & $\begin{array}{l}\text { Foram avaliadas potenciais barreiras técnicas que inviabilizariam a realização do projeto (ex: necessidade de utilização de } \\
\text { patentes existentes, infraestrutura indisponível, know-howinsuficiente)? Caso elas existam, essas foram contornadas? }\end{array}$ \\
\hline & & \multirow{8}{*}{ 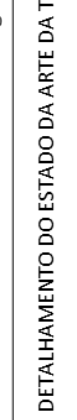 } & Técnico Go/Recycle & O conceito da tecnologia foi inicialmente definido? \\
\hline & & & Técnico Go/Recycle & $\begin{array}{l}\text { Os elementos/componentes básicos da tecnologia foram identificados e caracterizados de acordo com a necessidade } \\
\text { inicial (ex: identificação dos processos, operações unitárias, tendências)? }\end{array}$ \\
\hline & & & Go/Kill & A tecnologia tem se mostrado potencialmente replicável e escalável? \\
\hline & & & Go/Hold & $\begin{array}{l}\text { A infraestrutura, know-how, recursos humanos e financeiros necessários para se fazer a definição da rota básica de } \\
\text { processo estão disponíveis? }\end{array}$ \\
\hline & & & Go/Recycle & $\begin{array}{l}\text { Os aspectos de SMS das matérias-primas/produtos/ equipamentes foram considerados (riscos, periculosidade das } \\
\text { atividades e dos insumos que irão ser utilizados)? }\end{array}$ \\
\hline & & & Mercado Go/Recycle & Foi avaliada a necessidade de um parceiro para o desenvolvimento técnico do projeto? \\
\hline & & & Mercado Go/Recycle & Foi avaliada a necessidade de um parceiro para financiar o desenvolvimento do produto? \\
\hline & & & Go/Kill & $\begin{array}{l}\text { O desenvolvimento do projeto continua atrativo (interesse interno e/ou do parceiro, considerando também seu potencial } \\
\text { de mercado, aplicabilidade e custo)? }\end{array}$ \\
\hline
\end{tabular}

\subsection{Análise e discussão}

O estabelecimento do PDP permitiu o acompanhamento do estágio de desenvolvimento de cada projeto da organização, possibilitando não só uma melhor comunicação entre os agentes 
do processo, mas também a maior agilidade de análise e atuação em seus desafios. Tal benefício será potencializado a partir da integração do PDP à ferramenta utilizada para a gestão de projetos do CTNano, o que possibilitará, por exemplo, vincular o cronograma do projeto com as respostas dos gates. Além disso, é válido ressaltar os seguintes aspectos:

Balanceamento entre flexibilidade e estruturação e identificação de novas oportunidades de atuação ao longo do PDP

O FFE, equivalente à macroetapa de pré-desenvolvimento no modelo proposto, está inserido em um contexto de grandes incertezas. Em seus momentos iniciais foi adotada uma postura de grande flexibilidade e liberdade no processo, para incentivar a submissão de ideias. Essas seriam posteriormente agrupadas, avaliadas conjuntamente pela equipe de gestão e técnica, e selecionadas a partir da utilização de técnicas de gestão de portfólio. Entretanto, foi identificado que essa abordagem ainda não fornecia inputs significativos aos chamados "projetos preliminares", para os quais é necessário realizar testes iniciais, que fornecerão indícios sobre a viabilidade técnica do projeto ou ideia proposta. A partir de tal constatação, foi percebido que essa macrofase deveria ser modificada para suprir tais limitações. Além disso a partir do PDP confeccionado, foi possível identificar outras oportunidades de atuação conjunta das equipes de gestão e técnica ao longo do PDP, como, por exemplo, o auxílio na identificação e monitoramento de potenciais parceiros para os projetos do Centro.

\section{Auxílio na identificação de interfaces entre frentes de pesquisa}

Inserido em um cenário muito distinto do FFE, encontram-se as etapas que constituem o DP propriamente dito, nas quais há um número muito menor de incertezas, refletindo em sua maior previsibilidade, viabilidade de estruturação e formalização. Diante disso, se ressalta a contribuição do modelo proposto na determinação dos momentos adequados para a ocorrência de interfaces entre as frentes de pesquisa. Como exemplo, pode-se citar a identificação prévia dos momentos em que a frente transversal de pesquisa do CT que fornece todo o suporte às questões de SMS deve atuar. Tal interface está ilustrada na Figura 4, no gate "SMS".

Acompanhamento e determinação de uma atuação particularizada de acordo com as demandas identificadas em cada tipo de projeto

O DT é um tema complexo e não amplamente discutido na literatura da área de gestão como o FFE e DP. Esse trabalho trouxe uma abordagem que trata o DT como um conjunto de etapas do PDP que contém características tanto do FFE quanto do DP, como respectivamente: a presença de diversas incertezas e complexidades advindas do FFE e que ainda necessitam de serem trabalhadas no DT - como por exemplo, a definição da aplicação foco da tecnologia -; e a possibilidade de utilização do sistema stage-gate, não em sua forma tradicional como no DP, mas sim à luz do modelo stage-gate para DT de Cooper (2007), de modo mais flexível. Portanto, para adotar tal abordagem, foram analisados diversos aspectos relativos às aplicações tecnológicas que estavam sendo desenvolvidas tais como: o DT era focado na realização de melhoria incremental em tecnologia já existente?; na exploração de outra aplicação a partir de uma plataforma tecnológica?; ou no desenvolvimento de uma tecnologia disruptiva? Portanto, dependendo do que estiver sendo construído, as etapas do DT poderão agregar características mais semelhantes às do FFE ou DP. O que influencia diretamente no grau de flexibilidade dessas etapas e, consequentemente, nos métodos e técnicas adotados.

Outro elemento que respalda ainda mais a necessidade de se ter um acompanhamento e atuação particularizada principalmente durante o DT, consiste no fato de que uma tecnologia por si só não constitui o produto final que será entregue nas mãos do consumidor. Portanto, há decisões técnicas e de mercado críticas tomadas no DT, que irão direcionar a estratégia de utilização dessa tecnologia e consequentemente podem afetar seu desempenho no mercado. 
Vale ressaltar a contribuição trazida pela integração de elementos da metodologia TRA no PDP, de forma a auxiliar no direcionamento de questões técnicas para cada estágio de desenvolvimento, bem como reforçando o conteúdo dos marcos e etapas propostas. Ainda assim, é visto que há práticas utilizadas na operacionalização da metodologia TRA que poderiam ser mais profundamente analisadas e potencialmente incorporadas à realidade do Centro, tais como: o plano de maturação tecnológica, TRA Review Plan, e a metodologia utilizada para a identificação dos elementos tecnológicos críticos utilizados por DoE (2011).

Por fim, diante dos benefícios e limitações presentes no sistema confeccionado, acredita-se que durante sua implantação continuarão sendo identificadas oportunidades para adequá-lo cada vez mais à realidade do CTNano. Contudo, dado que já foram sinalizados avanços positivos em relação à contribuição do PDP, espera-se que esses sejam potencializados a partir de sua operacionalização efetiva, haja visto que o processo gerencial objetiva auxiliar na ocorrência de inovações de forma planejada e sistemática, em um ambiente naturalmente marcado por muitas incertezas e riscos, mas que justifica todo o esforço necessário diante de sua enorme capacidade de contribuição e geração de impacto no ecossistema de inovação.

\section{CONCLUSÃO}

O presente trabalho visou contribuir para o desenvolvimento de nanotecnologias em um contexto singular no Brasil. Para tal, a partir do estudo e interpretação dos desafios e incertezas de um Centro de Tecnologia em Nanomateriais, bem como se utilizando das literaturas de desenvolvimento de novos produtos e tecnologias e de TRLs, foi construído um PDP que pudesse nortear o andamento de projetos atuais e novos.

Durante sua construção foram consideradas peculiaridades do processo de desenvolvimento das tecnologias do Centro, para que se pudesse alcançar o resultado final almejado para esse trabalho: a construção de um PDP alinhado à realidade e às necessidades do CTNano. Acredita-se que a partir dessa sincronia poderão ser obtidas contribuições significativas para o desenvolvimento das tecnologias do Centro, sua profissionalização e a sistematização da inovação com nanomateriais. $\mathrm{O}$ intuito é mitigar riscos relativos ao processo, melhorar sua gestão e controle, bem como promover a diminuição do time to market das tecnologias.

Por se tratar de uma proposta inicial é necessário que esta seja melhorada continuamente a partir dos resultados e feedbacks. Por fim, espera-se que esse trabalho-base sirva de referência para iniciativas semelhantes no contexto de organizações que lidem com tecnologias portadoras de futuro para a economia do País, contribuindo para o aumento da frequência em que são inseridas novas tecnologias e produtos no mercado e, consequentemente, gerando mais valor para o desenvolvimento local.

A autora R.G.R agradece a Fundação de Amparo a Pesquisa de Minas Gerais (FAPEMIG), pelo apoio financeiro recebido para apresentação e publicação deste artigo.

\section{REFERÊNCIAS}

COOPER, R. G. Managing Technology Development Projects. IEEE Engineering Management Review, v. 35, n. 1, 2007. p. 67-76.

COOPER, R. G. Your NPD Portfolio May Be Harmful to Your Business's Health. PDMA Visions. v 29, n. 2, p. 22-26, 2005. 
COOPER, R. G. Stage-gate systems: A new tool for Managing new products. Business Horizons. v. 33, p. 44-54, maio. 1990.

DoE G 413.3-4A. Technology Readiness Assessment Guide. US Department of Energy, Washington, 2011. Disponível em: <https://www.directives.doe.gov/directivesdocuments/400-series/0413.3-EGuide- 04a>. Acesso em: 31 ago. 2016.

EESA. Technology Readiness Levels Handbook For Space Applications. 2008. Disponível em: $<$ https://artes.esa.int/sites/default/files/TRL_Handbook.pdf>. Acesso em: 07 set. 2016.

KHURANA, A.; ROSENTHAL, S. R. Towards Holistic "Front End" in New Product Development. Journal of Product Innovation Management, v. 15, p. 57-74, 1998.

KOEN, P.A. et al. PDMA Toolbook for New Product Development. New York: John Wiley and Sons, 2002. p. 2-35.

LUX RESEARCH. Statement of Findings: Sizing Nanotechnology's Value Chain. Sumário executive, outubro 2004, p. 31.

NOLTE, W. L.; KENNEDY, B. C.; DZIEGIEL, R. J. Technology Readiness Level Calculator. In: NDIA SYSTEMS ENGINEERING CONFERENCE. 2003. Disponível em:< www.dtic.mil/ndia/2003systems/nolte.ppt>. Acesso em: 01 out. 2016.

PISCOPO, M. R. et al. O Setor Brasileiro de Nanotecnologia: Oportunidades e Desafios. In: XXXVII ENCONTRO DA ANPAD, 2013, Rio de Janeiro. Anais... 2013.

PUGH, S. Total design: integrated methods for successful product engineering. Harlow: Addison Wesley, 1991.

ROBERTS, E. B. Managing Invention and Innovation. Research Technology Management, v. 31, n. 1, p.11-29,1988.

ROZENFELD, H. et al. Gestão de desenvolvimento de produtos: uma referência para melhoria de processo. São Paulo: Saraiva, 2006.

SILVA, J. C. T. Tecnologia: conceitos e dimensões. In: XXII ENCONTRO NACIONAL DE ENGENHARIA DE PRODUÇÃO - ENEGEP, 2002, Curitiba. Anais... 2002. (CD- ROM).

THOMAS, R. J. New Product Development: managing and forecasting for strategic success. New York: John Wiley \& Sons, 1993.

US National Nanotechnology Initiative. The National Nanotechnology Initiative StrategicPlan, 2007. Disponível: <nano.gov/default/files/pub_resource/nni_strategic_plan_2007.pdf $>$. Acesso em: 31 ago. 2016.

BELLIVEAU P.; GRIFFIN A.; SOMERMEYER S. The Pdma Toolbook 1 For New Product Development. Product Development \& Management Association, 2004. 480 p.

SALERNO, M. S. et al. Innovation processes: Which process for which project? Technovation, v.35, p.59-70, 2015. 